\title{
Electrical breakdown detection system for dielectric elastomer actuators
}

\author{
Michele Ghilardi (1,2), James J.C. Busfield (1,2), Federico Carpi (3) \\ (1) School of Engineering and Material Science, Queen Mary University of London, UK \\ (2) Materials Research Institute, Queen Mary University of London, UK \\ (3) Department of Industrial Engineering, University of Florence, Italy
}

\begin{abstract}
Electrical breakdown of dielectric elastomer actuators (DEAs) is an issue that has to be carefully addressed when designing systems based on this novel technology. Indeed, in some systems electrical breakdown might have serious consequences, not only in terms of interruption of the desired function but also in terms of safety of the overall system (e.g. overheating and even burning). The risk for electrical breakdown often cannot be completely avoided by simply reducing the driving voltages, either because completely safe voltages might not generate sufficient actuation or because internal or external factors might change some properties of the actuator whilst in operation (for example the aging or fatigue of the material, or an externally imposed deformation decreasing the distance between the compliant electrodes). So, there is the clear need for reliable, simple and cost-effective detection systems that are able to acknowledge the occurrence of a breakdown event, making DEA-based devices able to monitor their status and become safer and "selfaware". Here a simple solution for a portable detection system is reported that is based on a voltage-divider configuration that detects the voltage drop at the DEA terminals and assesses the occurrence of breakdown via a microcontroller (Beaglebone Black single-board computer) combined with a real-time, ultra-low-latency processing unit (Bela cape an open-source embedded platform developed at Queen Mary University of London). The system was used to both generate the control signal that drives the actuator and constantly monitor the functionality of the actuator, detecting any breakdown event and discontinuing the supplied voltage accordingly, so as to obtain a safer controlled actuation. This paper presents preliminary tests of the detection system in different scenarios in order to assess its reliability.
\end{abstract}

Keywords: Actuator, Dielectric Elastomer, Electrical Breakdown, Safety Mechanism

\section{INTRODUCTION}

Dielectric Elastomer Actuators (DEAs) are an emerging class of soft and lightweight actuators on the brink of leaving research labs to enter the mass market. This new class of actuators present a unique combination of a series of attractive properties, such as light weight, large strains, low cost of raw materials, high energy density, no energy required to hold a static position, noise- and heat-free operation, high strains, high efficiency and, despite their soft nature, high response speed [1-5]. Since their appearance in the scientific panorama, several research groups have performed extensive research work in order to facilitate their transition to the market as soon as possible. Many devices have been developed at different size scales and for different potential applications, such as optics, microfluidics and soft robotics, as has also been reported in several review papers [4], [5].

The underlying working principle of DEAs is relatively simple, as they can be considered rubbery capacitors made up of a deformable dielectric membrane coupled with compliant, stretchable electrodes. When an electric field is induced in the soft dielectric material by applying a voltage between the electrodes, the generated Maxwell stress causes the rubbery dielectric membrane to increase in surface and reduce in thickness [1]. The resulting actuation effect can be used to realize disparate kinds of devices.

Electrical breakdown of DEAs is an issue that has to be carefully addressed when designing systems based on this novel technology, since the driving electric fields required to obtain significant actuation are quite high (in the order of 100 $\mathrm{V} \mu \mathrm{m}^{-1}$ ) and they are close to the breakdown strength of the dielectric material. The risk for electrical breakdown often cannot be completely avoided by simply reducing the driving voltages, either because completely safe voltages might not generate sufficient actuation or because internal or external factors might change some properties of the actuator whilst in operation. For instance, the presence of defects or foreign bodies in the dielectric membrane, or the application of 
external forces to the soft actuator inducing localized deformations, and thus changes in the electric field, which could lead to early breakdown.

Unless the used DEA constitutive material and structure (namely the material and thickness of the electrodes) present a self-healing behaviour [6], the breakdown is a 'catastrophic' event, indicating that it is irreversible and destructive resulting in the formation of a narrow breakdown channel between the electrodes [7]. Catastrophic breakdown in solids is electrically power driven and, while it can be initiated by different mechanisms, it always results eventually in a thermal runaway involving melting, carbonization and/or vaporization of the dielectric material along the breakdown channel and the electrode material locally. This is caused by having high electric-field leakage currents in the dielectric material causing an increase of temperature, which causes an increase of conductivity of the medium. This, in turn, leads to a further increase in the leakage currents and, after a certain threshold, this process develops a positive feedback loop that quickly leads to thermal runaway and consequently to the formation of a permanent low-resistivity breakdown path between the electrodes.

When this happens, the electric field cannot reach a high enough level to achieve significant actuation anymore and the DEA loses its functionality.

When operating DEAs in a controlled lab environment and breakdown occurs, the high voltage power supply is usually switched off manually by the operator in order to stop applying the voltage to the broken DEA. A fast disconnection is needed to avoid the risk for damaging the electronics (which is driving a short-circuited load) and the risk that the DEA catches fire (due to thermal heating induced by the high current passing through the newly formed conducting path connecting the electrodes). This manual switching-off operation is incompatible with scenarios where the operator cannot or should not intervene, such as when the DEA has to operate unsupervised.

While there have been studies on predicting the occurrence of breakdown in DEAs [8], there is still a clear need for reliable, simple, portable and cost-effective detection systems able to acknowledge the occurrence of a breakdown event, enabling DEA-based devices able to monitor their status in order to make the systems safer and "self-aware".

\subsection{Breakdown detection system principle}

In this work, two possible practical scenarios for a DEA's dielectric breakdown event were identified. They are here defined as Channel BreakDown (CBD) and Tear BreakDown (TBD).

In the case of $\mathrm{CBD}$, an electrically conductive channel forms between the two electrodes of the DEA through the dielectric layer in correspondence of one (or more) breakdown spot/s and the DEA, from an electrical point of view, changes from a substantially capacitive load to a substantially resistive load. In the case of TBD, the same as for the case of CBD happens, but then the elastic energy stored in the elastomer (formed as a consequence of the large pre-stretch) causes a tear to grow from the breakdown spot, completely ripping the DEA, which, from an electrical point of view, further turns into an open circuit. Before getting ripped apart and opening its circuit's branch, the DEA undergoing TBD presents an electrically conductive path for a certain time window in which it is electrically equivalent to a DEA undergoing CBD.

In this work a simple portable system to monitor the occurrence of electrical breakdown of planar circular DEAs was developed. The system adopts a high-ratio voltage divider in parallel to the actuator and a control algorithm (implemented within a microcontroller) capable of determining in real time whether the actuator has undergone dielectric breakdown. The principle underlying this detection method is described below.

As shown in Figure 1, a resistive voltage divider was placed in parallel to the DEA (which can be electrically approximated to be a capacitor). The two resistances of the divider had a ratio of the order of $10^{3}$.

For any driving voltage, most of the current flows from the amplifier through the resistive branch forming the voltage divider.

When the DEA undergoes CBD, a low-resistance path establishes through the dielectric elastomer, and most of the current from the amplifier gets diverted from the resistive branch to the DEA branch, which now has a lower resistance. In this situation, the monitoring signal across $\mathrm{R}_{2}$ becomes significantly smaller than the voltage applied by the amplifier to the DEA's terminals and this fact can then be used as a good indicator of breakdown.

In the case of TBD the same as in the case of CBD happens but, after the DEA gets ripped apart, this behaves electrically as an open circuit and the current goes back to flow in the resistive branch, with the monitoring signal behaving not differently than in the situation with a non-broken DEA. The detection of breakdown in the case of TBD using this method has then to happen within the time window between the occurrence of the breakdown itself and the complete tearing of the DEA. We define this as the 'time-to-tear interval'.

CBD can then be thought of as a particular case of TBD with an infinitely long time-to-tear interval, making it easier to be detected. 


\section{NORMAL}

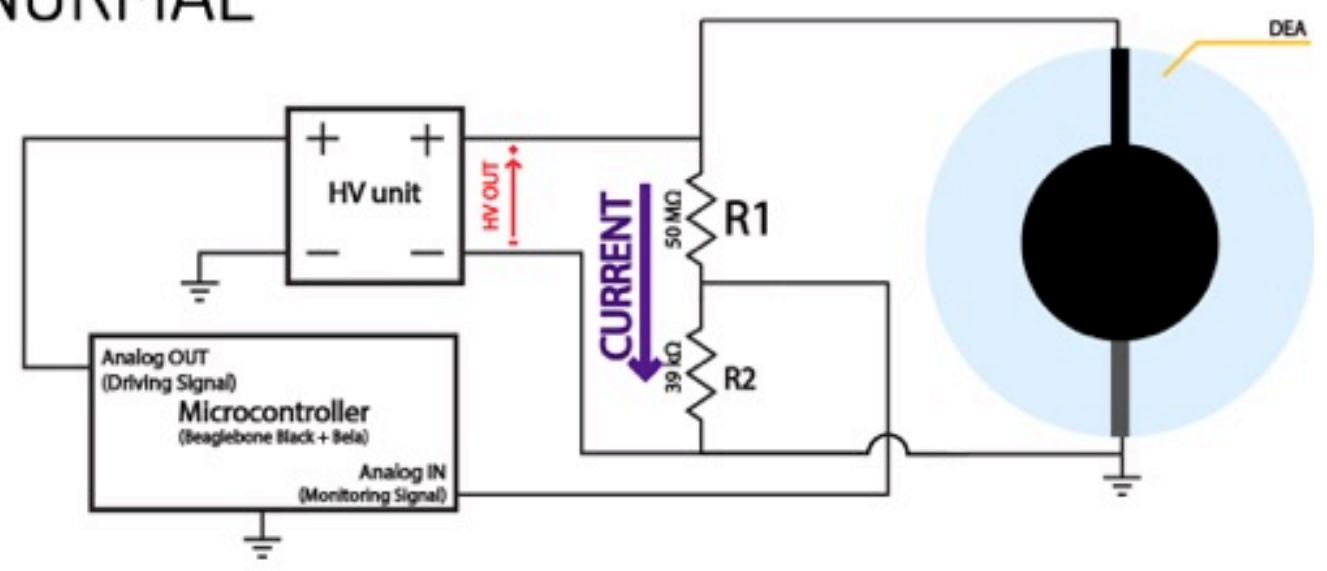

\section{$\mathrm{CBD}$ (detection time window)}

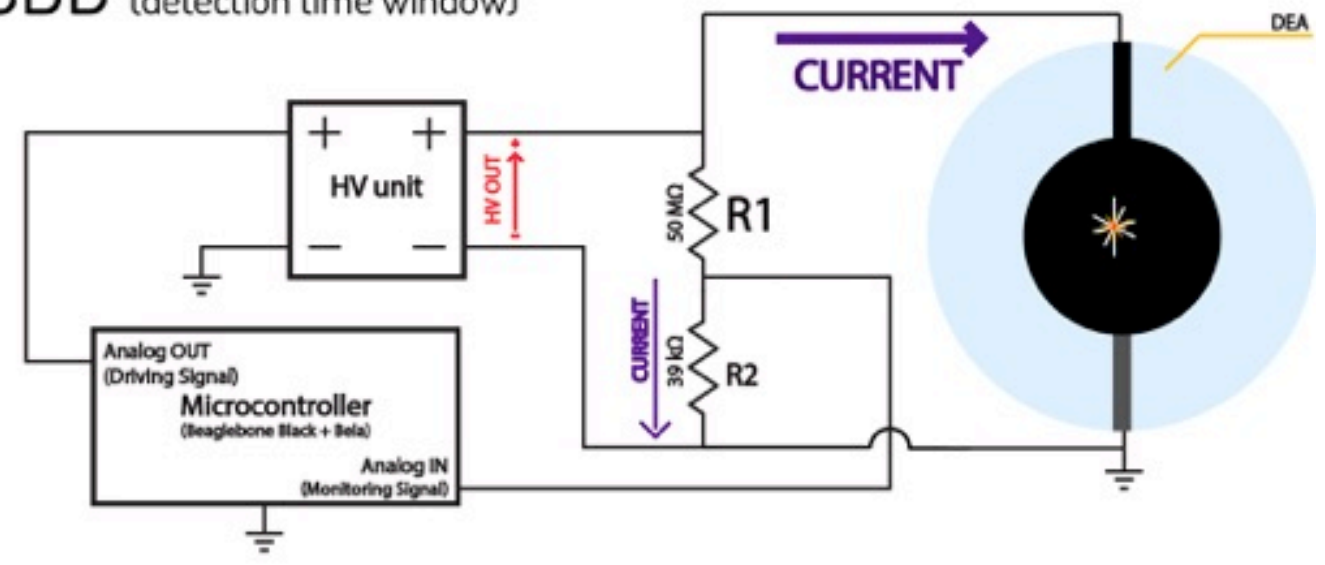

\section{TBD (after CBD)}

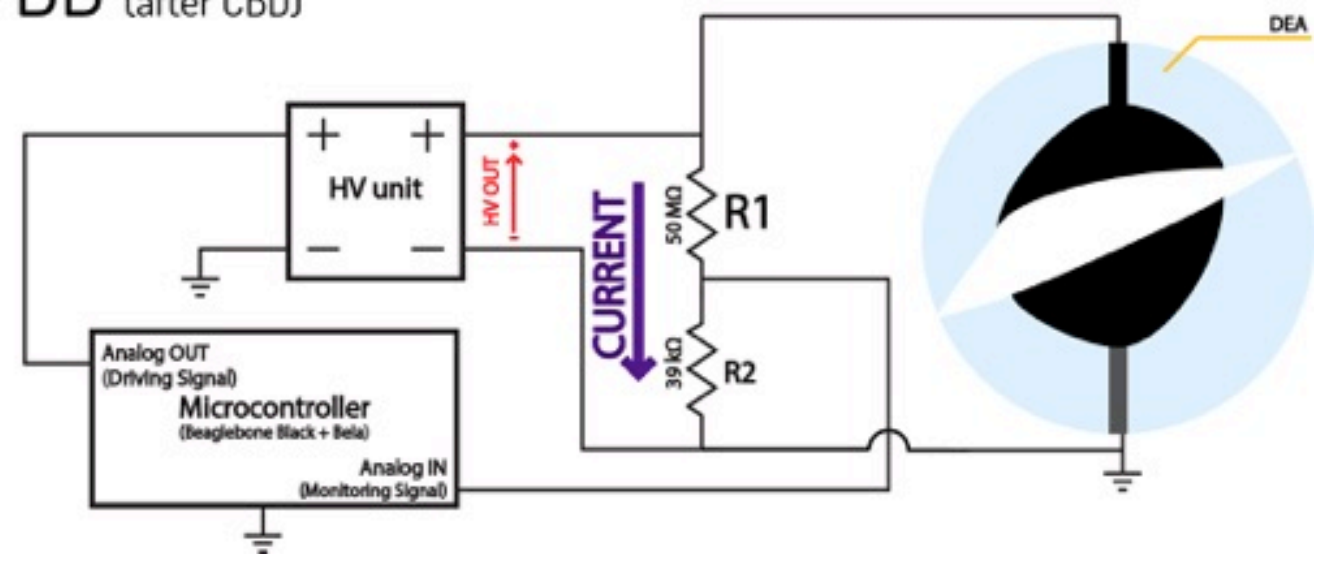

Figure 1 Schematic of the system used and the three possible scenarios: under normal operating conditions almost all of the current from the HV unit goes through the voltage divider's circuit branch, being the DEA a capacitive load; when breakdown is triggered and a low resistance path is formed across the DEA's membrane a Channel BreakDown (CBD) occurs with most of the current being diverted through the newly formed channel; when eventually tears propagate from the breakdown spot(s) and the DEA gets ripped apart, a Tear BreakDown (TBD) occurs and the current goes back to flow into the voltage divider's circuit branch. 
A breakdown detection system based on the described principle that works in a TBD case will then work for CBD as well without further modifications.

The only limitation of using such a breakdown detection principle without further considerations is that, in the case of self-healing electrodes, it could lead to the halting of the healing effect prematurely. When using some self-healing electrodes [9], it is believed that the initial stages of the thermal runaway could lead to the burning of the electrode material surrounding the edge of the breakdown channel, such that the electrical pathway through the dielectric elastomer is prevented and therefore the functionality of the DEA is restored. By using the breakdown detection system proposed in this work, which disconnects the voltage as soon as the monitoring voltage drops, the healing process could then be stopped before it has the chance to finish and thus the detection system would address the occurrence of breakdown even though the device could have recovered its functionality afterwards (if it was left to finish the healing process by maintaining the voltage applied). This limitation will be addressed in future developments of the work presented here, which therefore explicitly excludes DEAs with self-healing behaviour.

\section{MATERIALS AND METHODS}

\subsection{Samples used}

The DEA samples used for this study were prepared by equibiaxially pre-stretching the 3M VHB4905 acrylic elastomer film, using a custom-built stretcher, and sticking them to laser-cut acrylic frames to hold the film prestretched. Two prestretch ratios were tested: 3.5 and 4 times the original diameter (250\% and $300 \%$ increase, respectively). Circular electrodes and connection tracks were then realized by brush-painting carbon grease (MG Chemicals carbon grease) using stencils. Electrical contacts were realized with conductive copper tape and the conductive tracks painted with the same carbon grease used for the DEA's electrode (Figure 2).

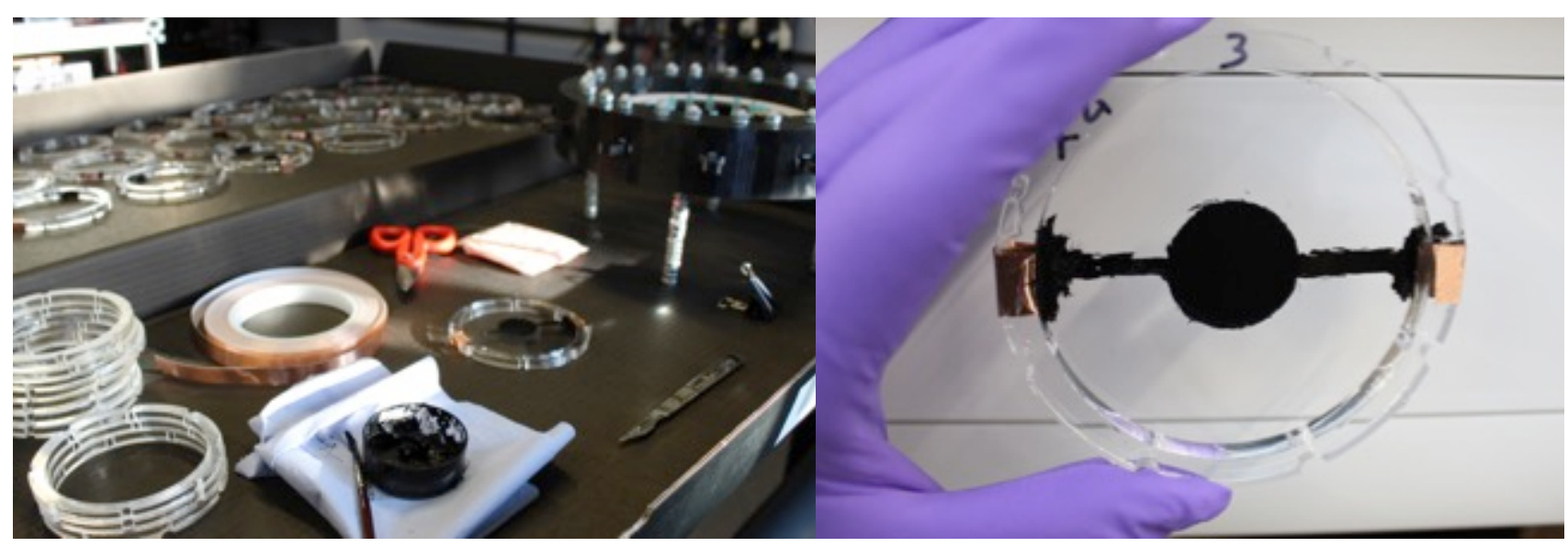

Figure 2 Left: materials and tools used to manufacture the samples; right: a sample DEA ready to be tested.

\subsection{Test rig}

The hardware control system used to test and implement the detection algorithm consisted of a laptop connected to a Beaglebone Black single-board microcontroller [10] paired with a Bela shield [11]. The latter is a low latency embedded system for real time processing developed at Queen Mary University of London. The system was programmed in C++ and was used both to generate the DEA control signal from one of the analog outputs and to read and store the analog signal from the voltage divider with a frequency of 22050 samples per second (sampling time of $45.36 \mu$ s) and a 16-bit resolution $(62.5 \mu \mathrm{V}$ vertical resolution). Data were processed with Matlab R2012b for non-live processing.

The signal generated by the analog output was fed to a High Voltage (HV) AC/DC amplifier (model 615-10-H-CE, Trek Inc., USA), which amplified it by a factor of 1000 . The HV output was applied to a custom made voltage divider connected to the DEA, and the monitoring signal measured across the smaller resistor of the voltage divider was sent 
back to one of the Bela's analog inputs, as shown in Figure 1. The resistance values were chosen in such a way that their sum was in the order of tens of $\mathrm{M} \Omega \mathrm{s}$, their ratio was about 1000, and the resulting output voltage did not saturate the analog inputs of the Bela shield. As the Bela's maximum analog output voltage when powered via USB is $4.86 \mathrm{~V}$ and the Bela's maximum analog input voltage is $4.096 \mathrm{~V}$, the maximum output of the HV amplifier then was $4.86 \mathrm{kV}$, which had to correspond to a maximum monitoring signal smaller than $4.096 \mathrm{~V}$ in order not to saturate the Bela's analog input. The values chosen for the resistances were $R_{1}=50 \mathrm{M} \Omega$ and $R_{2}=39 \mathrm{k} \Omega$, which corresponded to a voltage division by a factor of 1283. Consequently, the maximum voltage of $4.86 \mathrm{kV}$ applied to the DEA corresponded to a maximum monitoring signal of $3.788 \mathrm{~V}$.

\subsection{Breakdown detection system algorithm}

In an ideal system and under normal operating conditions (non-broken DEA), the monitoring signal would be equal to the driving signal after applying a gain correction parameter that takes into account the scaling factor due to the voltage divider.

When breakdown occurs, the current gets diverted into the broken DEA circuit branch and the monitoring signal's value read by the voltage divider drops significantly. If the difference between the driving signal and the monitoring signal is then calculated for each sample it can be said that this difference is ideally equal to zero when the DEA is not broken, while when the difference becomes greater than zero it means that breakdown has occurred.

Doing so would work without further considerations in an ideal, noise-free system. The noise that comes with the monitoring signal, being not negligible especially when using high-voltage low-cost components, makes the difference fluctuate around zero while the non-broken DEA operates. Without any proper correction, any fluctuation would trigger a false positive breakdown detection. This issue was addressed as described below.

The amplitude of the peak-to-peak noise read on the monitoring input was estimated, assessing that its value was around $4 \%$ of the maximum driving voltage $(4.86 \mathrm{kV})$. So, a detection threshold was defined as follows: when the difference between the monitoring signal and the driving signal exceeded a breakdown threshold equal to $5 \%$ of the maximum driving voltage the algorithm identified the occurrence of a breakdown event.

While this strategy would work straightforwardly for CBD, the situation is more complicated for TBD. Indeed, when TBD occurs, the signal difference exceeds the threshold value only for a short time, which is only during the time-to-tear interval, while the tears are propagating from the breakdown spots and the broken DEA acts as a low-resistance path for the current. Once the DEA gets completely ripped apart and the connection between the electrodes through the dielectric elastomer is interrupted, the current goes back to flow into the voltage divider branch and the signal difference goes back to values lower than the defined threshold.

So, for TBD events it is necessary to ensure that the system is able to identify even the shortest time-to-tear interval, i.e. the system can detect the occurrence of breakdowns without false positive or false negative detections. So, in other words, it is necessary to assess whether the shortest time-to-tear interval is longer than the sampling rate of the system.

\subsection{Assessment of the shortest time-to-tear interval}

In order to identify the shortest time-to-tear interval tests and verify that it was longer than the sampling rate of the system, the following tests were performed on a range of DEA samples that were prepared and tested. These included eight DEAs made of VHB4905 stretched by a factor of 3.5 and eight DEAs made of VHB4905 stretched by a factor of 4. High pre-stretch ratios were used because these correspond to a higher elastic modulus for the stretched elastomer and thus a faster tear growth rate. With faster tear growth rates the time-to-tear interval for TBD get shorter, and this scenario complicates the breakdown detection. Indeed, the detection gets more difficult because it has to happen before the end of the time window, when the DEAs get physically ripped apart opening their circuit branch.

The test consisted in applying a voltage ramp from 0 to $4.86 \mathrm{kV}$ in 10 seconds and then holding the maximum voltage for 1 second before dropping it to $0 \mathrm{~V}$. The monitoring signal read via the voltage divider during the application of the voltage ramp was acquired for later processing.

The time-to-tear interval for a TBD event was defined as follows. Let us consider the instant $t_{1}$ when the difference between the driving and monitoring signals exceeds the detection threshold defined above, and let us also consider the 
instant $t_{2}$ when the difference drops below the same threshold and stays there until the end of the applied voltage ramp. The time-to-tear interval was defined as the time difference $t_{1}-t_{2}$.

It is worth noting that the so-obtained time-to-tear interval is just an estimate based on the arbitrary criteria set above.

To avoid false positive breakdown detections and obtaining wrong estimates of the time-to-tear interval, the events in which the signal difference was greater than the detection threshold only for one sample were ignored. Indeed, in that case the event were not related to the occurrence of an actual breakdown event but, rather, to random fluctuations. Considering that the sampling time of the chosen microcontroller was $45.36 \mu$ s, the system was able to detect breakdown events when the time-to-tear interval was longer than $90.72 \mu \mathrm{s}$.

Using the definition just described, the average, standard deviation and minimum value of the detected time-to-tear intervals were determined for each of the two groups of the differently prestretched samples.

\subsection{Breakdown detection system testing}

The detection algorithm was tested with eight DEAs made of VHB4905 stretched by a factor of 3.5 and eight DEAs made of VHB4905 stretched by a factor of 4.

The test consisted in applying a driving signal, consisting of a biased sinusoidal wave with its amplitude growing at each cycle, defined by Eq. (1):

$$
\text { Driving signal }=A(t) \frac{(1+\sin (2 \pi f t+\theta))}{2}
$$

where $A(t)$ was a time-dependent amplitude (which was varied in steps from 0 to the maximum voltage of $4.86 \mathrm{kV}$ adding $1 / 3^{\text {rd }}$ of the maximum voltage every $2 \pi$ increase in phase), the frequency was set to $f=0.5 \mathrm{~Hz}$, and the phase offset was set to $\theta=3 / 2 \pi$.

While the driving signal was applied to a DEA, the monitoring voltage from the voltage divider was recorded and processed by the microcontroller. Eventually the electric field in the dielectric material reached a value high enough to trigger the breakdown. The event was detected by the system, which immediately sets the driving voltage to $0 \mathrm{~V}$. 


\section{RESULTS AND DISCUSSION}

\subsection{Time-to-tear intervals}

The average, standard deviation and minimum values of the time-to-tear intervals are reported, for each set of samples, in Table 1, along with the driving voltage value at which breakdown was detected. All the tests that were carried out with highly stretched DEAs, resulted in TBD events. The presented results show that the minimum time-to-tear interval was $9.4 \mathrm{~ms}$, proving that the microcontroller used, having a sampling time of $45.36 \mu \mathrm{s}$, was well suited for detecting even the quickest TBD event. The significant variability in the duration of the time-to-tear intervals was likely due to the fact that this value can be influenced by unpredictable factors, such as the spatial position on the DEA where the breakdown spots form (and the subsequently triggered tears start propagating), the "ripping pattern" that the tears form while doing so, as well as the presence of defects and foreign particles in the elastomer and the electrode.

Even though the relatively low number of samples tested limited the statistical significance of the data, it can be noticed that the average time-to-tear interval for the DEAs was lower for the higher prestretch, as expected. There are anyway significant outliers in both groups (as the high standard deviations also suggest), as also shown for example in Figure 3 where a DEA with a higher pre-stretch shows a time-to-tear interval longer than one second.

Table 1 Values of the detected time-to-tear intervals and the breakdown voltages for the two groups of DEAs equibiaxially pre-stretched by different ratios (x3.5 and x4). All the samples underwent TBD.

\begin{tabular}{|c|c|c|}
\hline $\begin{array}{l}\text { SAMPLE \# } \\
(\text { stretch } \times 3.5)\end{array}$ & $\begin{array}{c}\begin{array}{c}\text { Time-to-tear } \\
\text { interval }\end{array} \\
{[\mathbf{s}]} \\
\end{array}$ & $\begin{array}{c}\text { Breakdown } \\
\text { voltage } \\
{[\mathrm{kV}]}\end{array}$ \\
\hline 1 & 0.0306 & 3.8232 \\
\hline 2 & 0.2193 & 3.6381 \\
\hline 3 & 0.4557 & 3.5919 \\
\hline 4 & 0.1254 & 3.7601 \\
\hline 5 & 1.6082 & 3.5581 \\
\hline 6 & 0.3934 & 3.2733 \\
\hline 7 & 0.8906 & 3.4502 \\
\hline 8 & 0.6954 & 3.3759 \\
\hline AVERAGE & 0.5523 & 3.5589 \\
\hline ST. DEV & 0.5141 & 0.1869 \\
\hline MINIMUM & 0.0306 & 3.2733 \\
\hline
\end{tabular}

\begin{tabular}{|c|c|c|}
\hline SAMPLE \# & $\begin{array}{c}\text { Time-to-tear } \\
\text { interval }\end{array}$ & $\begin{array}{c}\text { Breakdown } \\
\text { voltage }\end{array}$ \\
(stretch x4) & {$[\mathbf{s}]$} & {$[\mathbf{k V}]$} \\
\hline 1 & 0.0094 & 3.7018 \\
2 & 1.1797 & 3.9514 \\
3 & 0.3210 & 3.6087 \\
4 & 0.1453 & 4.1823 \\
5 & 0.3019 & 4.2941 \\
6 & 0.5786 & 4.2570 \\
7 & 0.6405 & 4.1144 \\
8 & 0.2569 & 4.3462 \\
\hline AVERAGE & $\mathbf{0 . 4 2 9 2}$ & $\mathbf{4 . 0 5 7 0}$ \\
ST. DEV & $\mathbf{0 . 3 6 7 4}$ & $\mathbf{0 . 2 7 7 0}$ \\
MINIMUM & $\mathbf{0 . 0 0 9 4}$ & $\mathbf{3 . 6 0 8 7}$ \\
\hline
\end{tabular}



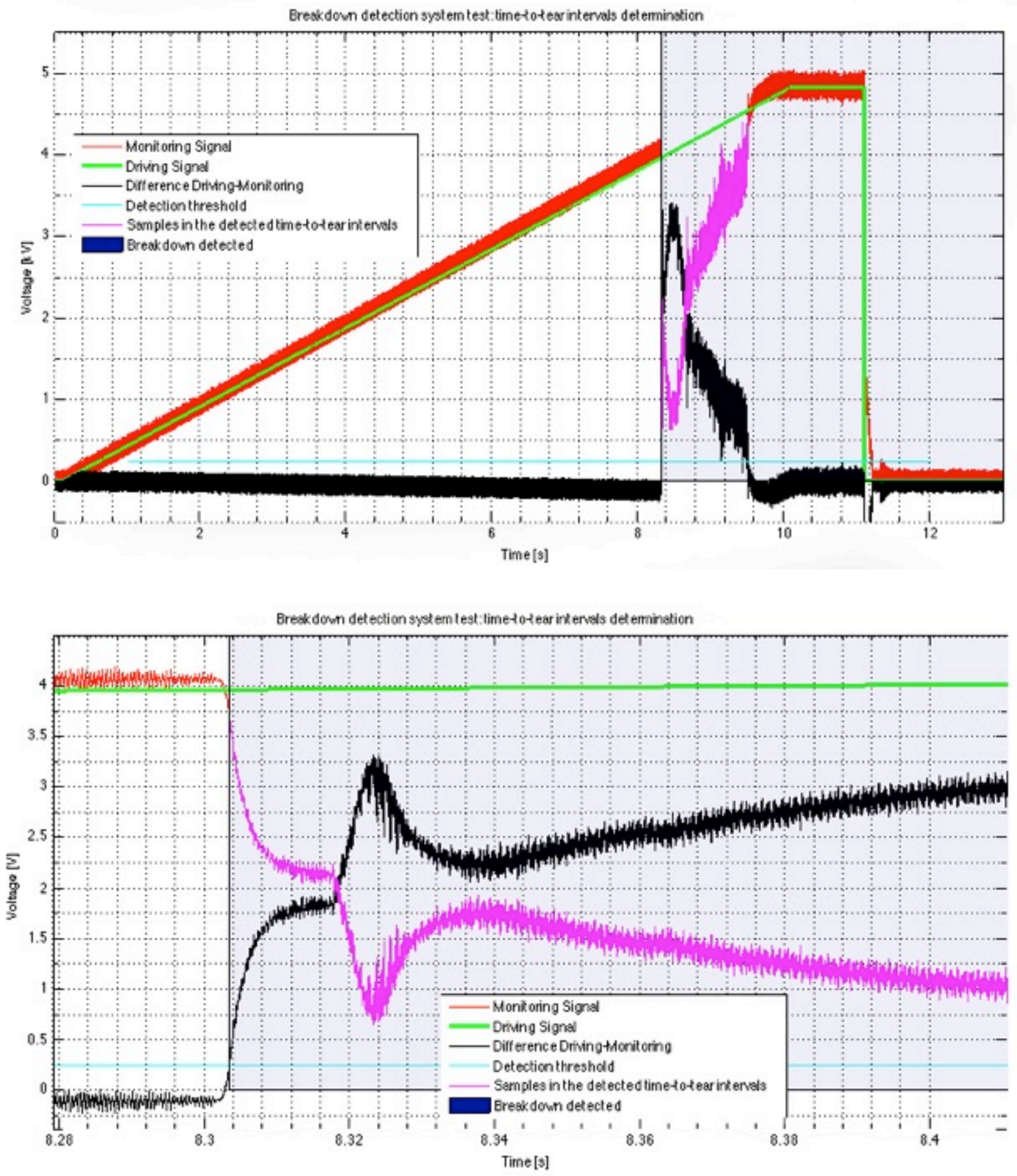

Figure 3 Determination of the time-to-tear interval for a VHB4905 sample equibiaxially stretched 4 times its original diameter. This particular sample took a relatively long time to get ripped apart, hence a long time-to-tear interval, despite its high pre-stretch value. In the graphs the simple concept on which the detection algorithm relies can be appreciated: when the difference between the driving and the monitoring signals (black line) exceeds the empirically defined breakdown threshold (light blue line) for more than one sample, the breakdown event is detected (shaded area) and the time-to-tear interval starts getting tracked (red line turning pink) until the difference drops below the threshold again (pink line turning back to red). This can be better appreciated in the bottom graph which shows a zoom in on the data near the breakdown event from the above graph. For a colour version of the figure see the online paper. 


\subsection{Breakdown detection testing}

The designed breakdown detection system worked for all the samples tested, quickly detecting the breakdown events successfully and disconnecting the applied voltage. There were no false positive or false negative detections. The typical data acquired during the tests looks like those in Figure 4, where the growing sinusoidal driving signal (green line) is overlapped to the noisier monitoring signal from the voltage divider (red line). Their difference is plotted in black and, when this exceeds the detection threshold (light blue line) set at 5\% of the maximum voltage, breakdown was assessed by the algorithm which set the driving signal to zero.
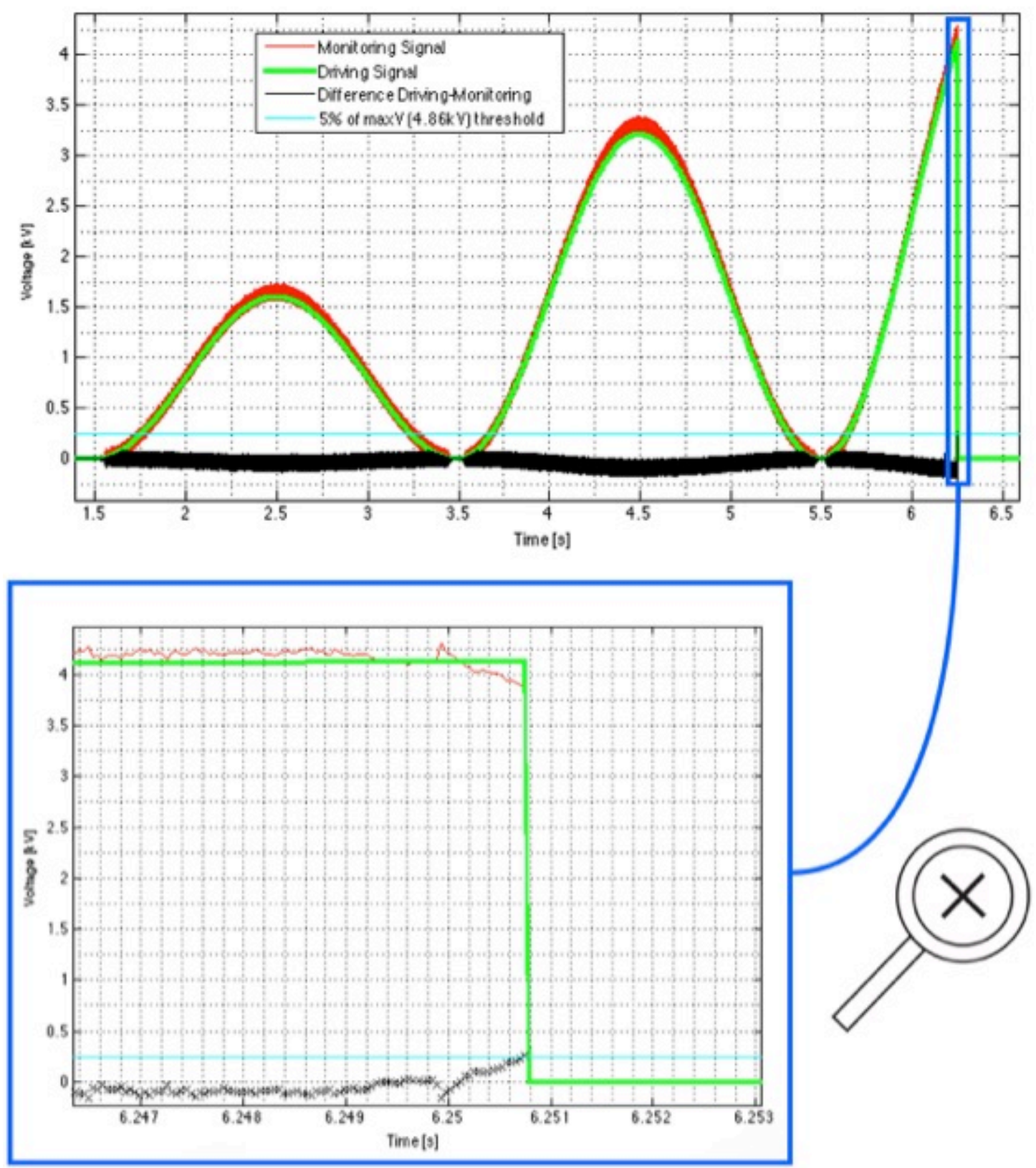

Figure 4 Example of a breakdown detection. The ramping-up sinusoidal driving signal (green line), the monitoring voltage signal (red line) and the difference between them (black line) are co-plotted. Once the difference signal exceeds the 5\% threshold line (light blue) breakdown was assessed and the driving voltage was set to zero, as is highlighted in zoomed-in graph at the bottom. For a colour version of the figure see the online paper. 
The breakdown voltages and the type of breakdown occurred for each sample are reported in Table 2 . It can be appreciated that, unlike the tests with the ramping voltage where all the events led to TBD, here 7 out of 16 samples underwent just CBD and, thanks to the detection system that quickly switches off the applied HV, the process was stopped without further evolving into TBD. This was an indication that, since with the detection system the current flows through the dielectric elastomer for a very short time after the breakdown was detected, the thermal runaway effect was reduced and does not lead to triggering a tear big enough to propagate. Such a reduction of the thermal runaway leads to a significant reduction of the risk of fire.

Table 2 Values of the breakdown voltage for the two groups of DEAs stretched by different ratios, and type of breakdown event tat occurred for each of them (TBD or CBD).

\begin{tabular}{|c|c|c|}
\hline SAMPLE \# & $\begin{array}{c}\text { Breakdown } \\
\text { voltage } \\
\text { (stretch x3.5) }\end{array}$ & $\begin{array}{c}\text { Breakdown } \\
\text { type }\end{array}$ \\
\hline 1 & 3.8960 & TBD \\
2 & 4.2760 & CBD \\
3 & 4.5980 & TBD \\
4 & 4.0650 & TBD \\
5 & 3.5080 & TBD \\
6 & 4.0100 & CBD \\
7 & 4.4090 & CBD \\
8 & 4.5690 & TBD \\
\hline AVERAGE & $\mathbf{4 . 1 6 6 4}$ & \\
ST. DEV & $\mathbf{0 . 3 7 0 4}$ & \\
MINIMUM & $\mathbf{3 . 5 0 8 0}$ &
\end{tabular}

\begin{tabular}{|c|c|c|}
\hline SAMPLE \# & $\begin{array}{c}\text { Breakdown } \\
\text { voltage } \\
\text { (stretch x4) }\end{array}$ & $\begin{array}{c}\text { Breakdown } \\
\text { type }\end{array}$ \\
\hline 1 & 3.6480 & CBD \\
2 & 4.2940 & TBD \\
3 & 4.1110 & TBD \\
4 & 3.5270 & CBD \\
5 & 4.4240 & CBD \\
6 & 3.9970 & TBD \\
7 & 3.7640 & CBD \\
8 & 4.1320 & TBD \\
\hline AVERAGE & $\mathbf{3 . 9 8 7 1}$ & \\
ST. DEV & $\mathbf{0 . 3 1 5 8}$ & \\
MINIMUM & $\mathbf{3 . 5 2 7 0}$ &
\end{tabular}

\section{CONCLUSIONS AND FUTURE DIRECTIONS}

The preliminary data collected during this work suggest that the simple and inexpensive breakdown detection system for DEAs presented here might be effective to detect breakdown events without false negative or false positive detections. It is a solution that could easily and effortlessly be implemented in systems using DEAs in order to make them capable of self-assessing their malfunctioning by just adding a few cheap electronic components to the system and a few lines of code to the control algorithm.

Further systematic testing is necessary to assess this solution's effectiveness to a wider extent (requiring a higher number of validation tests) and in different scenarios (such as testing different prestretch ratios and different materials). Moreover, other scenarios that could be tested include using different microcontroller units (for example a slower, but cheaper and easier to program, Arduino unit) and cheaper, more compact and portable HV units (such as an EMCO Qseries unit), as well as systems with different levels of noise that could interfere with the detection.

Furthermore, as already mentioned above, such a breakdown detection system could lead to problems if used for DEAs with self-healing electrodes, due to the fact that it could stop the healing process before its completion, assessing the DEA's failure too quickly. So, a modification of the system aimed at making it usable also with self-healing DEAs is desirable.

Moreover, it would also be interesting to record the breakdown events using an high speed camera and acquiring the monitoring signal in synchrony, in order to assess the delay between when the breakdown is visibly triggered and when it gets electrically detected by the system. 


\section{ACKNOWLEDGMENTS}

This project has received funding from the European Union's Horizon 2020 research and innovation programme under the Marie Skłodowska-Curie grant agreement No 641822-MICACT (MSCA-ITN-2014-ETN).

\section{REFERENCES}

[1] Pelrine R., Kornbluh R., Pei Q, Joseph J., "High-speed electrically actuated elastomers with strain greater than 100\%", Science, 287:836-839 (2000).

[2] Carpi, F., De Rossi, D., Kornbluh, R., Pelrine, R.E., \& Sommer-Larsen, P., [Dielectric elastomers as electromechanical transducers: Fundamentals, materials, devices, models and applications of an emerging electroactive polymer technology], Elsevier (2008).

[3] Carpi F., Bauer S., De Rossi D., "Stretching dielectric elastomer performance", Science 330, 1759-1761 (2010).

[4] P. Brochu, Q. Pei, "Advances in Dielectric Elastomers for Actuators and Artificial Muscles", Macromol. Rapid Communications 31 (1) 10-36, (2010).

[5] S. Rosset, H. R. Shea, "Small, fast, and tough: Shrinking down integrated elastomer transducers", Appl. Phys. Rev. 3(3), 031105, (2016).

[6] F. B. Madsen, L. Yu, A. L. Skov, "Self-Healing, High-Permittivity Silicone Dielectric Elastomer", ACS Macro Lett. 5(11), 1196-1200, (2016).

[7] L. A. Dissado, J. C. Fothergill, [Electrical Degradation and Breakdown in Polymers], IET Digital Library (1992).

[8] T. A. Gisby, S. Q. Xie, E. P. Calius, I. A. Anderson, "Leakage current as a predictor of failure in dielectric elastomer actuators", Proc. SPIE 7642, 764213-764213-11 (2010).

[9] S. Michel, B. T. T. Chu, S. Grimm, F. A. Nüesch, A. Borgschulte, D. M. Opris, "Self-healing electrodes for dielectric elastomer actuators", J. Mater. Chem. 22(38), 20736-20741 (2012).

[10] "BeagleBoard.org - beaglebone black". 25 Feb 2017, https://beagleboard.org/black

[11] "Bela.io". 25 Feb 2017, http://bela.io/. 